\title{
URBAN SPRAWL ANALYSIS USING SHANNON'S ENTROPY AND FRACTAL ANALYSIS: A CASE STUDY ON TIRUCHIRAPPALLI CITY, INDIA
}

\author{
Kriti Rastogi ${ }^{1, *}$, Gaurav V. Jain ${ }^{1}$ \\ ${ }^{1}$ Space Applications Centre, Ahmedabad, Gujarat, India- (kritirastogi, gvj)@ sac.isro.gov.in
}

Commission V, SS: Infrastructure and Development Planning

KEY WORDS: Shannon's Entropy, Fractal Analysis, Box counting, Urban Sprawl

\begin{abstract}
:
Urban sprawl places a major role in urban growth management all over the world. Urban growth of the city is analysed for the effective planning and improving the socio-economic conditions for decision making and the development of the city. In this study, the urban sprawl of Tiruchirappalli city is analysed from 2006 to 2017 using data of Indian remote sensing satellites, Resourcesat1 and Resourcesat- 2 with multi spectral band (red, green and near-infrared). The temporal change for four-time periods is calculated using object based image analysis temporal inversion approach. From 2006 to 2017 there is an increase in total urban area from $58 \mathrm{~km}^{2}$ to $90 \mathrm{~km}^{2}$, resulting of $33 \%$ increment in 11 years. Shannon entropy is calculated for four-time periods and it shows that after $8 \mathrm{~km}$ from Central Business District (CBD), Shannon entropy is increasing and dispersion is high. The influence of roads on urban sprawl is calculated using buffer function analysis and the highest contribution among all the highways is by NH-67 and $\mathrm{NH}-210$.The fractal analysis of the urban area for four-time period is performed and fractal dimension is calculated. Fractal dimension helps in measuring the space filling extent and urban growth complexity and is used to characterise the compactness of urban pattern and regularities of urban boundaries. The fractal dimension of Tiruchirappalli increases from 1.584 in 2006 to 1.639 in 2017.
\end{abstract}

\section{INTRODUCTION}

Tamil Nadu is one of the most urbanised states in India with $48.5 \%$ of the state population living in urban areas as per the census of (2011). Tiruchirappalli being the fourth largest city of Tamil Nadu, shows major urban growth in past two decades. The increase in population and unplanned development of urban structures results into problems like lack of availability of resources, infrastructures, services and facilities. Hence, population is moving away from the dense towards outer parts of city in an unplanned manner leads to urban sprawl. One of the major factors of sprawling is industrialization, which contributes to the economy of the city. This drives people from other cities and towns to take benefit from the economy of the city. Understanding and identification of urban growth pattern and spatial and temporal changes in urban area; help in planning and development of infrastructures and facilities.

The industrial market of Tiruchirappalli is very well established. In 1964, with the advent of one of the largest engineering and manufacture industries of India in integrated power plant equipment manufacturer, Bharat Heavy Electrical Limited (BHEL), advancement in modern industrialization took place in Tiruchirappalli. Many other small scale and large scale industries were developed fulfilling the need of paper mills, sugar mills and cement mills etc. The government took the initiation towards the growth of Information Technology sector in the city by development of first IT Park called, ELCOT IT Park. It opened the door for many reputed MNC's to city and significant contribution to the economy of the city. The Indian Ordnance Factories Board set up two urban centres, towards the rural areas in the outer parts of the city. The dispersed development towards the rural areas along the highways in the vicinity of the city, leads to urban sprawl. The consequences of sprawling are change in land use and land cover of large agricultural fields, vacant lands and water bodies to urban areas that leads to many environmental problems (Radhakrishnan et.al., 2014). In simple words, the increase in the population in an area or city and lack of resources makes people to shift

defense bodies in the city namely, Ordnance Factory Tiruchirappalli (OFT) and Heavy Alloy Penetrator Project (HAPP). These industries are established in south eastern part of the city. These industries enabled Tiruchirappalli, to mark a significant place in the industrial market of the country. The city with major industries situated in eastern and southern part contributes the majorly to the urban development towards these directions.

The city is well connected to the nearby cities and states through roads, rail network and Airport facilities. Tiruchirappalli International Airport, being the second largest airport in Tamil Nadu after Chennai International Airport serving Tiruchirappalli and adjacent districts. It is located on NH-210 (Tiruchirappalli to Rameshwaram). The railway network of Tiruchirappalli has five branches which connect it to Chennai, Kanyakumari , Erode, Rameshwaram and Karaikal. This well connected railway station in central Tamil Nadu becomes important railway station in the southern railway network.

* Corresponding author 
Through this study we are trying to analyse the urban sprawl of the Tiruchirappalli city of Tamil Nadu. The urban change detection for four years is done using remote sensing and GIS techniques. The object based image analysis temporal inversion approach is used for calculating the temporal and spatial changes in past one decade. Shannon's Entropy is used to study the dispersion of urban area for four-time period. The study also attempts to find out the factors responsible for urban sprawl and quantify its influence on the sprawling of the city. Among many factors, roads are one of the important factors responsible of urban sprawls. The Buffer function analysis is used for calculating the rate of change of growth along the roads and to quantify the influence of highways on urban growth. The Pearson correlation coefficient is used for finding out the correlation of growth along roads and the entire city. The analysis of urban land use and land cover for four years is done by using fractal analysis of the urban areas.

\section{STUDY AREA}

Tiruchirappalli, also known as the Trichy, is the fourth largest city of Tamil Nadu State in India. It is geographically located at $10^{\circ} 44^{\prime} 46^{\prime \prime} \mathrm{N}$ to $10^{\circ} 52^{\prime} 46^{\prime \prime} \mathrm{N}$ latitude, $78^{\circ} 39^{\prime} 11^{\prime \prime} \mathrm{E}$ to $78^{\circ} 44^{\prime} 13$ " E longitude. The city is spread in area of $167 \mathrm{~km}^{2}$ area and its topography is flat with altitude of $88 \mathrm{~m}$ above sea level. Tiruchirappalli is situated on the banks of river Cauvery, which is a major source of water supply for the city. As per the census 2011, city has a total population of 916,857 in year 2011. Tiruchirappalli administration is governed by Tiruchirappalli Municipal Corporations. The civic administration of the city is divided into four zones Abhishekapuram (West), Ariyamangalam (East), Golden Rock (South) and Srirangam (North), which has in total 65 wards. Tiruchirappalli is a major educational hub with prestigious institutes like Indian Institutes of Management, National Institutes of Technology, Indian Institutes of Information Technology, Anna University and others. The city is well connected to the major cities and states through roads, rail network and Airport facilities. The major National Highways such as NH38, NH67, SH22, NH45 and NH-210 passes through the city and connect to nearby cities and towns. The industrial market of the city is very well established and gives major contribution to the economy of the city. The city has residential and commercial area spread in the northern and southern part of the city. The old part of the city is congested with houses.

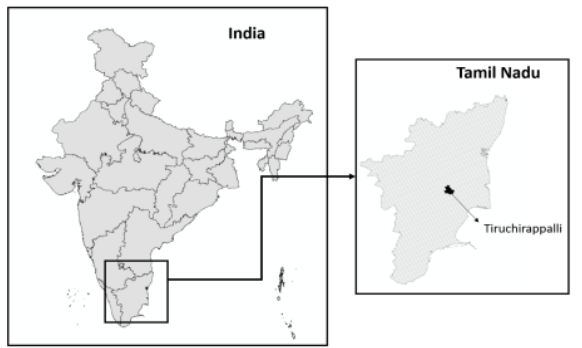

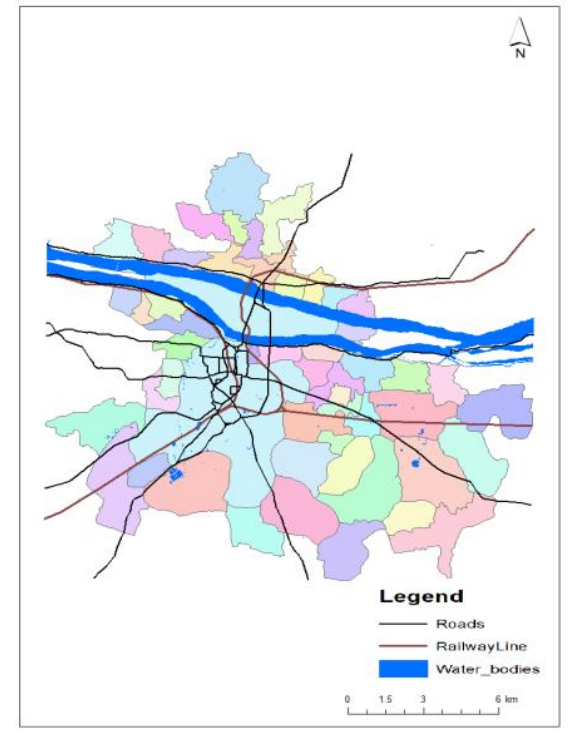

Figure 1. Study Area Location

\section{DATA USED}

The study used the data acquired by Indian remote sensing satellite (IRS) launched by Indian Space Research Organisation (ISRO). IRS-Resourcesat-1 and Resourcesat-2 satellites provides $5.8 \mathrm{~m}$ spatial resolution, three bands (Green, Red and Infrared) multi spectral images acquired by LISS -4 sensor. The multi temporal data for 2006 to 2017 is used with four scenes as shown in Table1. The geographical boundaries of the study area are obtained from the Natural Resource Database under National Natural Resource Management System (NNMRS) (ISRO 2005), mapped under Natural Resources Information System (NRIS) project corresponding to year 2005 at 1: 50,000 scale.

\begin{tabular}{|c|l|l|l|l|}
\hline $\begin{array}{c}\mathrm{N} \\
\mathrm{o}\end{array}$ & Satellite & Sensor & $\begin{array}{l}\text { Res } \\
(\mathrm{m})\end{array}$ & $\begin{array}{l}\text { Date of } \\
\text { Acquisition }\end{array}$ \\
\hline 1 & Resourcesat-2 & LISS 4 & 5.8 & $1 / 6 / 2017$ \\
\hline 2 & Resourcesat-2 & LISS 4 & 5.8 & $1 / 7 / 2014$ \\
\hline 3 & Resourcesat-1 & LISS 4 & 5.8 & $4 / 5 / 2010$ \\
\hline 4 & Resourcesat-1 & LISS 4 & 5.8 & $1 / 5 / 2006$ \\
\hline
\end{tabular}

Table1. Data Used 


\section{METHODOLOGY}

\subsection{Built-up Extraction and Change Detection}

Multi temporal remote sensing data is used for extracting the built up area of the study area. All the satellite images are coregistered using the geo-coded ortho-rectified Resourcesat-2 LISS-4 images. Using the visual interpretation technique water bodies were manually digitized.

Object based image analysis (OBIA) approach is applied using nearest neighborhood classification with hierarchical multi-resolution segmentation to extract the built up area of the city (Jain and Sharma, 2018). The object based temporal inversion is applied for change analysis of built-up area using eCoginition Developer 9.5 software (Trimble Documentation, 2015). The built up area of the subsequent year will be more than that of previous year. The built-up area of 2017 is used for extracting the built-up area of 2014, and the latter is used as an input for extracting the built-up area of 2010. At last for finding the change from year 2006 to 2010, 2010 built-up area is used as an input for 2006 data.

Multi- resolution image segmentation is applied on the four scenes with scale parameter 30 , weights of 0.7 and 0.3 for shape and compactness respectively. Training set of for four classes i.e. built-up area, built-up with vegetation, soil and vegetation is selected. The object based nearest-neighbor classification approach is applied using object features such as mean of all the layers, normalised vegetation index, standard deviation of each layer. The built-up area map generated for four years is mapped at the 1:25,000

\subsection{Spatial proximity analysis}

To derive the influence of roads in the urban sprawl, spatial proximity analysis is done. A multiple buffer ring of $250 \mathrm{~m}$ till the distance of $13000 \mathrm{~m}$ is created around Central business district (CBD), assuming it on the centre most part of the city. Shannon's entropy and urban area are calculated for each zone to do a comparative growth analysis over a period of time. A $500 \mathrm{~m}$ buffer along the major roads such as National Highways and State Highways is created along with $250 \mathrm{~m}$ buffer around the CBD. The intersection of these two buffers helps in studying the influence of roads on urban sprawl. A unique identity is given to each buffered road and urban area is calculated for each buffered area along the road for every time period to quantify the contribution of each road in urban growth over a period as shown in Figure 2.

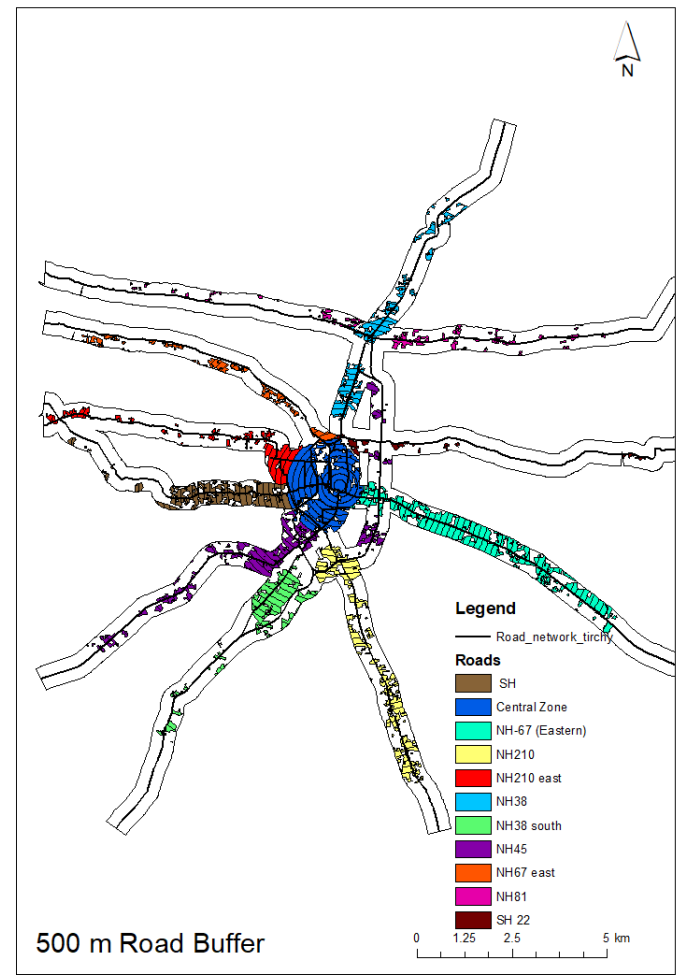

Figure 2. 500m buffer along the roads

Shannon's entropy $\left(H_{n}\right)$ is used to measure the degree of spatial concentration or dispersion of geophysical parameter among the $\mathrm{n}$ zones. It is given by equation (1)

$$
H_{n}=-\sum_{1}^{n} p_{i} \log \left(p_{i}\right)
$$

where, $\mathrm{P}_{\mathrm{i}}$, is the probability of variable occurring in the $\mathrm{i}^{\text {th }}$ zone and $\mathrm{n}$ is the total number of zones. (Yeh and $\mathrm{Li}, 2001$ ) The value of entropy varies from zero to $\log (n)$. The value of zero indicates that the distribution is very compact, and the value near to $\log (\mathrm{n})$ is highly disperse in nature. High value of entropy indicates occurrence of sprawl (Sudhira et.al., 2004). Relative Entropy, also called Shannon equitability index, has great importance in measurement of diversity and similarity for spatial related issue in the field of biology, urban studies (Yeh and Li, 2001). It is given by equation (2).

$$
R n=\frac{\sum_{1}^{n} p_{i} \log \left(p_{i}\right)}{\log (n)}
$$

\subsection{Fractal Analysis}

The theory of fractals is coined by Mandelbrot (B.B and Mandelbrot, 1983). Fractals are scale invariant, self-similar structures which can be expressed as the union of sets of each reduce copy of geometrically similar structure that forms the full set. Many natural objects such as clouds, coastlines, mountains having non smooth shapes shows fractal nature. The fractal nature of city is a valuable input for studying the characteristics of a city. Fractal dimension quantifies the 
fractal characteristics of the city (Batty and Longley, 1994). One of the methods of determining the fractal dimension is box counting method. Box counting method is mathematically defined as in equation (3), suppose that $F$ is a limited fractal figure on a plane. Cover $F$ with square grids which are built by boxes whose side length is $\delta$. The number of boxes intersecting on $F$ is $N$. If $N$ is satisfied with the power law

$$
N(\delta) \alpha \delta^{-D},
$$

then as $\delta \rightarrow 0$, the ratio of logarithm $D=\frac{\ln N(\delta)}{\ln \left(\frac{1}{\delta}\right)}$ , $D$ is defined as the box dimension of $F$ (Ye and Chen, 2001).

The fractal dimension is given by the slope of the straight line formed by plotting $\ln \mathrm{N}(\delta)$ and $\ln (1 / \delta)$, (known also as the Richardson-Mandelbrot plot (Falconer,1985). If the trend is linear, the observed object is assumed to be fractal ( $\mathrm{Ge}$ and Lin, 2009). For a 2-dimension image the value of slope is in the range between 1 and 2 which infer that it lies between the straight line and a line that is wiggly enough that it covers a 2- dimension space. The steeper the slope of the straight line, the more 'fractal' an object is and it gain complexity with the decrease in the size of the box.

The extracted vector data of built-up area for four years, 2006, 2010, 2014, and 2017 is converted into 2-state image with value 1 assigned for urban areas and 0 for non-urban areas. The corresponding image for four years is used for finding the fractal dimension of study area for each year. These four images are exported in MATLAB. The image size is padded to nearest $2^{\mathrm{n}} \times 2^{\mathrm{n}}$ image (for e.g. image of size $312 \times 200$ image is changed to the dimension of $512 \times 512$ ). Two type of box counting method are there for estimating the fractal dimension of the study area: (Benguigui et.al., 2000) one to fix the size of the largest box and other to have a variable size of the largest box covering the study area (Clark,1951). In this study, a fix size largest box is used for all the years for comparison purpose. We need to count the number of non-zero submatrixes divided by $2^{\mathrm{n}}$ using a recursive algorithm. Initially the image is sub-divided into four equal sub-images and the number of non -zero sub-matrixes of each sub-image divided by $2^{\mathrm{n}-1}$ is calculated. Their sum is the number of the superior image. The process is repeated till $n=1$, when no there is no submatrix left. (M. and Lin, 2009).

\section{RESULTS}

The urban area of the city is spread in total $58 \mathrm{~km}^{2}$ in 2006 was increased to $90 \mathrm{~km}^{2}$ in 2017 showing the built-up growth of $33 \%$ in past 11 years. During 2006 to 2010 per year, urban growth was found to be $4 \%$, which in 2010 to 2014 was $2.40 \%$ and in 2014 to 2017 decreased $2.26 \%$. The decrease in per year growth from 2006 to 2017 is due to occupancy of vacant land which is decreasing with urban sprawl the limited availability of resources. The urban sprawl from year 2006 to 2017 is shown in Figure 3.

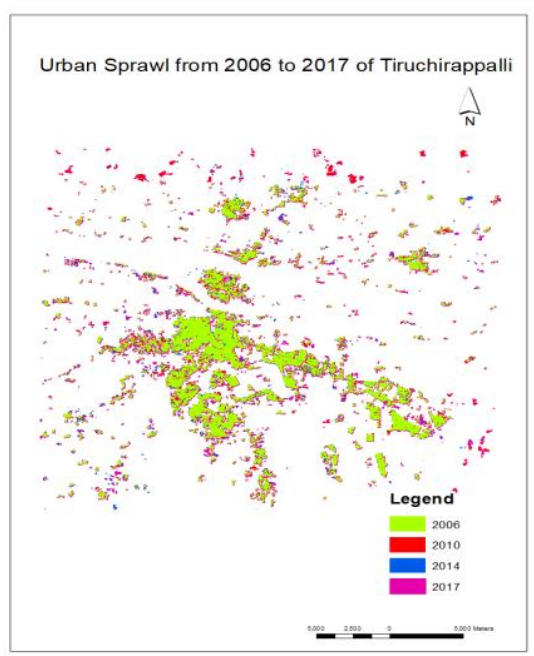

Figure 3. Urban Sprawl from year 2006-2017

Shannon's entropy was calculated from the built-up area for each individual zone $(n=52)$. The value of entropy for Tiruchirappalli for 2006 was 3.85 and for 2017 was 3.88 while $\log (\mathrm{n})$ for $\mathrm{n}=52$ value is 3.95 . The value of entropy is close to $\log (\mathrm{n})$ hence it shows high degree of dispersion in urban areas in the study area. The graph in Figure 4 shows that there is a disperse growth from 8 to $9 \mathrm{~km}$ from the city center and it became more dispersed as we move away from the city. The Relative Shannon's Entropy in urban area for each zone is calculated for four time periods and it shows a monotonically increasing behavior after $8 \mathrm{~km}$, hence the urban growth is observed in the outer part of the city.

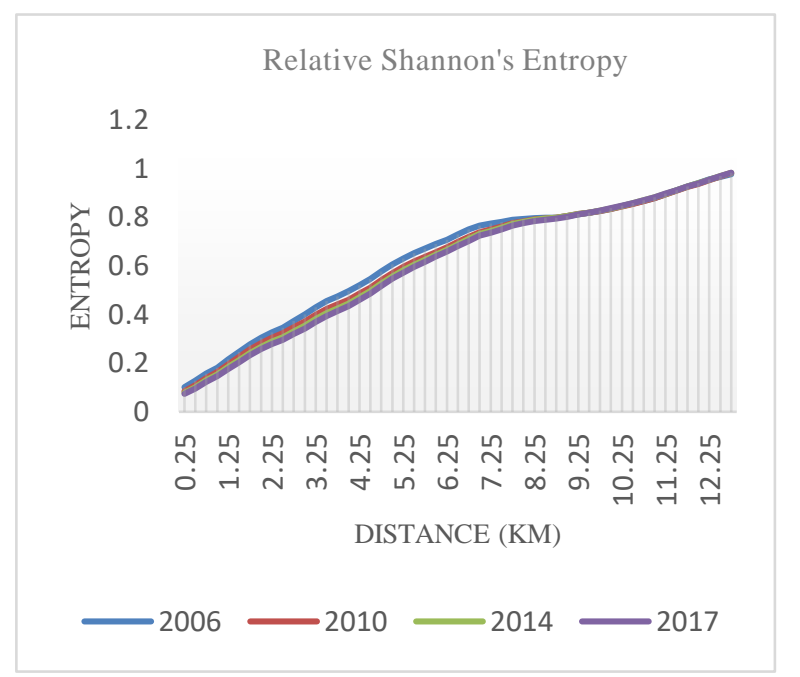

Figure 4. Zone wise Relative Shannon entropy from year 2006-2017

In Tiruchirappalli city, linear urban growth is observed along the Highways. The major National Highways such as NH-45, NH-45, NH67, SH-38 and NH-210 passes through the city and connect to nearby cities and towns. NH-45 connects the city with the capital of Tamil Nadu, Chennai. The city is connected to Rameshwaram through the NH-210 heading towards south. From the eastern side the NH-67 connects Tiruchirappalli to 
Thanjavur. The NH-38 directly connects the city to Madurai with the stretch of $135 \mathrm{~km}$.

A high degree of influence is established with Pearson correlation coefficient value 0.982 obtained between the growth along the roads and the urban growth of the city. Highways are major driving factor of urban growth. As per the results, the major urban development took place along the direction of NH-67 east. This highway has numerous industrial areas, educational institutes and other urban settlement. The second most influential road was NH-210 connecting Tiruchirappalli with Rameshwaram with many Information Technology centres established along it. It was found that the relative urban development of these areas in four time periods shows development along NH-67 east is less likely to grow more in future because it reaching to a saturation level as shown in Figure 5.

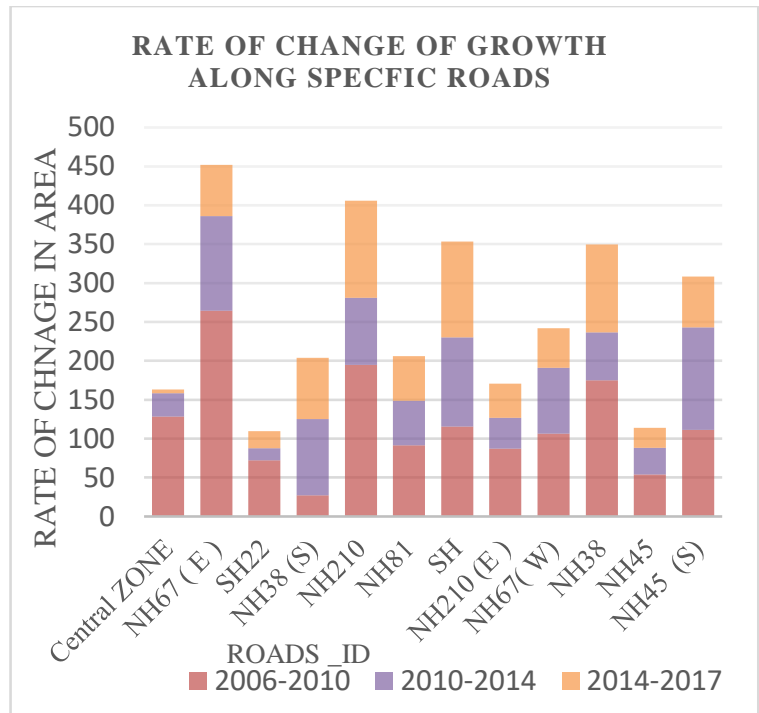

Figure5. Rate of change of urban area along the roads from 2006-2017

The fractal analysis of the urban area from 2006 to 2017 is done using box counting method. From Figure 6, Linear trend is found between the $\log (\delta)$ and $\log N(\delta)$, hence Tiruchirappalli city is fractal in nature. Fractal dimension is increasing from 1.5835 in 2006 to 1.613 in 2010 and to 1.628 in 2014 and 1.639 in 2017. This shows that the fractal dimension increases with time and with the increase in space filling extent of the city. The increment of 0.055 in fractal dimension from 2006 to 2017 is found. The local dimesion of farctal object is given by $-\frac{d}{d r} \ln (\delta)$, which gives the range of box counting dimension and its associated error range.The local dimension for 2006 is 1.5835 with an error range of $+/$ 0.27138 which is increased to 1.6948 with error range $+/$ 0.22216 in 2017. The change in local dimension of box counting method is within the error range. Hence the change fractal dimension is not that significant. This implies that the fractal dimension can be adopted to measure the space filling extent and spatial complexity of urban growth.
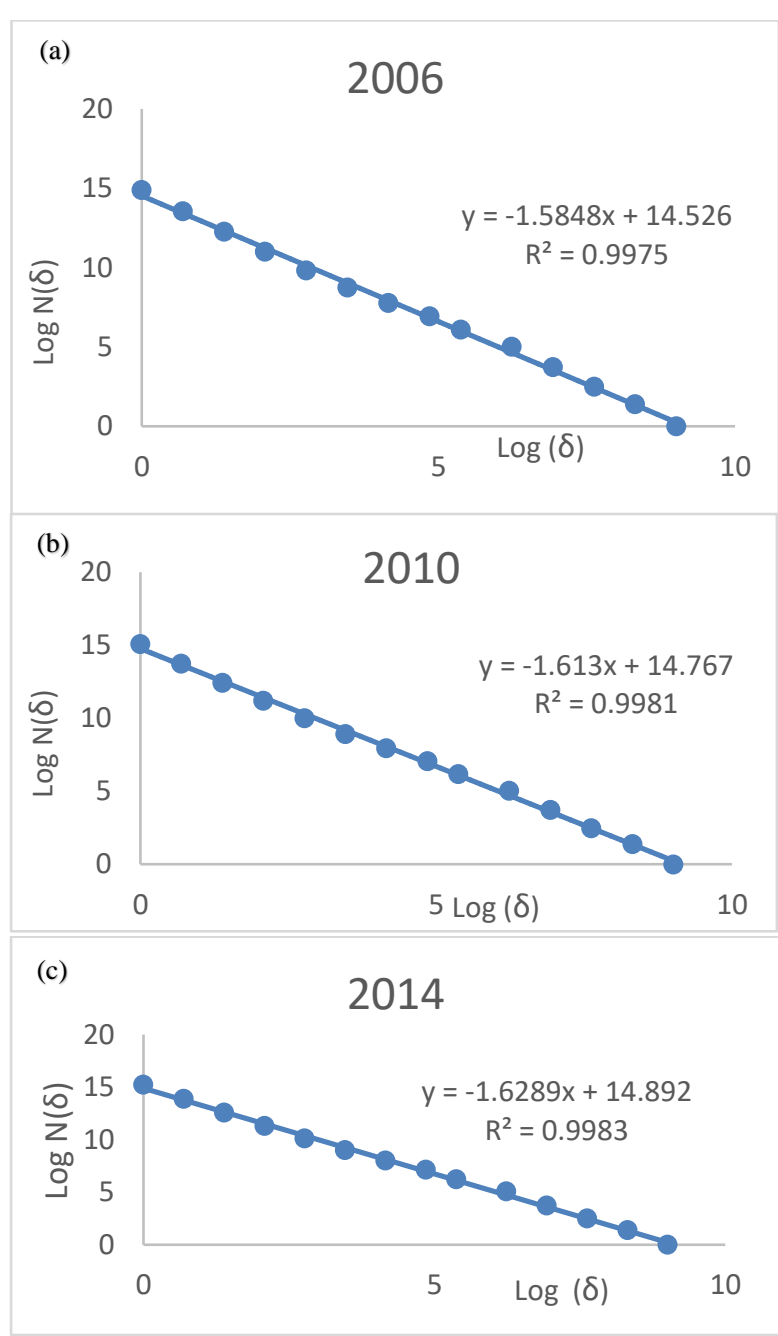

(d)

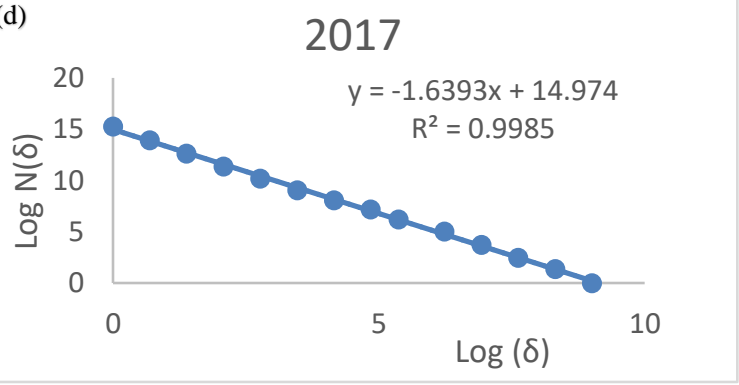

Figure6. Fractal dimension of urban area from year (a) 2006, (b) 2010, (c) 2014, (d) 2017

\section{CONCLUSION}

In this study, urban sprawl analysis of Tiruchirappalli city is analysed. Urban sprawl is observed at the outer part of the city.Using Shannon entropy, high dispersion is found after $8 \mathrm{~km}$ from Central Business District.Tiruchirappalli shows the linear urban growth. The city is expanding along the highways away from the dense urban patch. A high corelation is found between the growth along the roads and the urban growth of the entire city.Tiruchirappallii city is fractal in nature. The 
fractal dimension of the city increases with time and with increase in space filling extent of the city. The local farctal dimension of the city is within the error range. This study helps in understanding the urban growth pattern of Tiruchirappalli and the factors influencing in the urban growth.

\section{ACKNOWLEDGMENTS}

The author would like to thank Shri D.K. Das, Director Space Applications Centre for all the support and guidance during the course of study. We would like to express our sincere gratitude to Dr Raj Kumar, Shri Shashikant A. Sharma, Dr Markand Oza, Dr A S Rajawat and Dr B.S. Munjal for their valuable inputs.

\section{REFERENCES}

Batty M., Longley P., 1994. Fractal Cities: A geometry and Function. Academic Press London, U.K.

Benguigui L., Czamanski D., Marinov M., PortugaliY., 2000. When and Where is a City Fractal?. Environment and Planning and Design, Volume 2, Issue 4, 507-519, https://doi.org/10.1068/b2617.

Chen Y., Wang J., Feng J., 2017. Understanding the Fractal Dimension of Urban Forms through spatial entropy, Entropy. Volume 19, Issue 11,https://doi.org/10.3390/e19110600.

Clark, C. (1951). Urban Population Densities. Journal of the Royal Statistical Society. Series A (General), Volume 114, Issue 4, pp. 490-496. doi:10.2307/2981088.

ISRO. 2005. NNRMS standards, ISRO: NNRMS: TR: 112: 2005, Committee Report. Bangalore: Indian Space Research Organisation (ISRO), Department of Space, Government of India.

Jain G.V., Sharma S.A., 2018. Spatio-temporal analysis of urban growth in selected small, medium and large Indian cities. Geocarto International, doi:10.1080/10106049.2018 .1450450 .

M. Ge, Q. Lin, 2009. Realizing the Box-counting Method for Calculating Fractal Dimension of Urban Form Based on Remote Sensing Image. Geo-spatial Information Science, doi: 10.1007/s11806-009-0096-1, Volume 12, pp. 265-270.

Mandelbrot B.B, 1983.The Fractal Geometry of Nature, W.H. Freeman, San Francisco.

Radhakrishnan N., Eerni S.K., Kumar S., 2014. Analysis of Urban Sprawl Pattern in Tiruchirappalli City Using Applications of Remote Sensing and GIS. Arabian Journal for Science and Engineering, Volume 39, Issue 7, pp. $5555-5563$
Sudhira H. S., Ramachandra T. V., \& Jagadish K. S., 2004. Urban Sprawl: Metrics, Dynamics and Modelling Using GIS. International Journal of Applied Earth Observation Geoinformation, Volume 5, Isuue 1, pp. 29-39 https://.doi.org/10.1016/j.jag.2003.08.002.

Trimble Documentation. 2015. Trimble eCognition ${ }^{\circledR}$ Developer. Reference Book. Munich: Trimble Germany $\mathrm{GmbH}$.

Ye J., Chen B.Z., 2001. The application of the fractal theory in the city research. Urban Planning Forum 4, pp. 38-42.

Yeh A.G., X. Li., 2001. Measurement and Monitoring of Urban Sprawl in a Rapidly Growing Region Using Entropy, Photogrammetric Engineering and remote sensing, Volume 67, Issue 1, pp. 83-90. 